\title{
Distributed and dynamic strain sensing with high spatial resolution and large measurable strain range
}

\author{
LI Zhang, ${ }^{1,2, *}$ (1) Zhisheng Yang, ${ }^{2}$ (1) NAchum Gorbatov, ${ }^{1}$ Roy Davidi, ${ }^{1}$ Malak Galal, ${ }^{2}$ (]) \\ Luc ThÉVenaz, ${ }^{2}$ (i) AND Moshe Tur ${ }^{1}$ \\ ${ }^{1}$ School of Electrical Engineering, Tel-Aviv University, Tel-Aviv, Israel \\ ${ }^{2}$ Institute of Electrical Engineering, EPFL Swiss Federal Institute of Technology, CH-1015 Lausanne, Switzerland \\ ${ }^{*}$ Corresponding author: li.zhang@epfl.ch
}

Received 22 April 2020; revised 10 July 2020; accepted 21 July 2020; posted 22 July 2020 (Doc. ID 395922); published 4 September 2020

\begin{abstract}
A distributed and dynamic strain sensing system based on frequency-scanning phase-sensitive optical time domain reflectometry is proposed and demonstrated. By utilizing an RF pulse scheme with a fast arbitrary waveform generator, a train of optical pulses covering a large range of different optical frequencies, short pulse width, and high extinction ratio is generated. Also, a Rayleigh-enhanced fiber is used to eliminate the need for averaging, allowing single-shot operation. Using direct detection and harnessing a dedicated least mean square algorithm, the method exhibits a record high spatial resolution of $20 \mathrm{~cm}$, concurrently with a large measurable strain range $(80 \mu \varepsilon, 60$ demonstrated), a fast sampling rate of $27.8 \mathrm{kHz}$ (almost the maximum possible for a $55 \mathrm{~m}$ long fiber and 60 frequency steps), and low strain noise floor $(<1.8 \mathrm{n} \varepsilon / \sqrt{\mathrm{Hz}}$ for vibrations below $700 \mathrm{~Hz}$ and $<0.7 \mathrm{n} \varepsilon / \sqrt{\mathrm{Hz}}$ for higher frequencies). (๑) 2020 Optical Society of America
\end{abstract}

https://doi.org/10.1364/OL.395922

Provided under the terms of the OSA Open Access Publishing Agreement Phase-sensitive optical time-domain reflectometry ( $\Phi$-OTDR) [1] is an important Rayleigh-based dynamic fiber-optic sensing (DDFS) technique that has established itself as an effective and highly sensitive means to probe standard single-mode fibers. This technique measures the interference of waves reflected from random intrinsic density variations along the fiber, illuminated by a coherent propagating input pulse. While initially used to detect (and locate) only the occurrence of induced perturbations along the fiber, the technique has been recently upgraded to quantitatively measure strain [2-7]. Since a local strain change linearly varies the phase of light reflected from the strain-affected scatterers, key to proper retrieval of the strain variations along the fiber is phase demodulation of the backscattered light. Different phase demodulation schemes, such as detection using a $3 \times 3$ coupler [4], phase carrier modulation [5] and I/Q demodulation [8], were proposed.

In most applications, particularly in SHM, a short spatial resolution (SR) associated with a large dynamic range is required, since the strain change in a structure can be large and local, e.g., for the detection of cracks. Much effort has been carried out to further push the SR. Systems using linear frequency modulated interrogating pulses have been implemented in $\Phi-O T D R$, pushing the SR to the submeter range $(90 \mathrm{~cm} \mathrm{[3]}$ and $34 \mathrm{~cm}$ [9]). Yet another $\Phi$-OTDR implementation based on perfect periodic autocorrelation (PPA) coding claims DDFS with a demonstrated SR of $10 \mathrm{~cm}$ [10]. All the systems use coherent detection, where the coherent length of the laser may limit the sensing range, and the demonstrated strain range is limited to a magnitude of hundreds of $n \varepsilon$ (nanostrain). Instead of using phase demodulation schemes, the phase change of the scattered light was retrieved (still coherently) from an equivalent spectral shift of the Rayleigh interference pattern, using frequency-scanned interrogating pulses [11], which enabled static quantitative measurement of strain/temperature changes. Based on this interrogating scheme, a fast frequency-scan scheme has been demonstrated by a direct modulation of the laser current [12] using direct detection, albeit, with limited linearity over wide scan ranges. Alternatively, a single-shot measurement has been demonstrated using chirped-pulse $\Phi-O T D R[13]$. However, the SR in these systems is not shorter than a few meters.

In this Letter, a direct-detection distributed and dynamic strain system is proposed and demonstrated with high SR and large measurable strain range, based on frequency-scanning $\Phi-O T D R . ~ A ~ h i g h-f r e q u e n c y$, high-speed RF pulse modulation scheme is used, offering: (i) practically zero latency in the frequency scanning, enabling a fast scanning speed; and (ii) high extinction ratio for the employed narrow optical pulses, to avoid any spatial crosstalk, thereby ensuring proper high SR. A backscatter-enhanced fiber was employed as the sensing fiber, providing a backscattered signal strong enough to eliminate the need for averaging, thereby making the measurement sampling rate limited only by the fiber length and the number of frequency scanning steps. A post-processing algorithm based on a previously developed least mean square algorithm [14] is employed, allowing the minimization of the number of frequency scanning steps. Benefiting from all these design features, our direct-detection $\Phi$-OTDR system simultaneously 


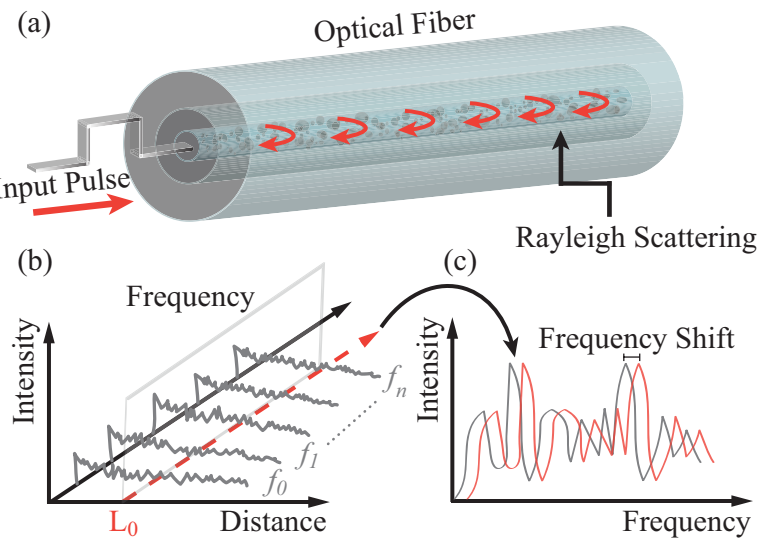

Fig. 1. Principle of frequency-scanning $\Phi$-OTDR: (a) conceptual view of interrogation in $\Phi$-OTDR; (b) intensity of Rayleigh backscattered traces for the different scanning optical frequencies; (c) Rayleigh spectra of reference and measurement showing the strain-induced frequency shift at position $L_{0}$.

exhibits a record combination of a high $S R$ of $20 \mathrm{~cm}$, a maximum measurable strain of $80 \mu \varepsilon$, together with a fast sampling rate of $27.8 \mathrm{kHz}$ and a strain noise floor of $<0.7 \mathrm{n} \varepsilon / \sqrt{\mathrm{Hz}}$ for frequencies $>700 \mathrm{~Hz}$.

The principle of our interrogating technique is based on a frequency-scanning $\Phi$-OTDR [14], illustrated in Fig. 1. Optical pulses are sent into the fiber, and the intrinsic density variations of the fiber, frozen during the fiber manufacturing process, cause Rayleigh backscattering [Fig. 1(a)]. The positionresolved Rayleigh spectral patterns of the fiber are obtained by scanning the frequency of the interrogating pulse [Fig. 1(b)]. In close analogy to OFDR-based strain interrogation [15], the measured frequency shift, Fig. 1(c), is proportional to the strain change between the corresponding Rayleigh spectra.

The scheme of the system is depicted in Fig. 2. A distributed feedback laser with a standard $1 \mathrm{MHz}$ linewidth is used as a light source. An electro-optic modulator (EOM) biased in carriersuppressed mode, driven by a fast AWG (M8195A 65 GSa/s, courtesy of Keysight/Israel), together with a tunable sharp-edge optical filter (filter 1, bandwidth of $45 \mathrm{GHz}$ covering the full scanning range) and an erbium-doped fiber amplifier (EDFA1), comprise the subsystem that generates short and strong pulses with a high extinction ratio (see below for more details). The amplified pulses are then launched into the fiber under test (FUT) through a circulator. The Rayleigh-backscattered light from the fiber is pre-amplified by EDFA2, whose amplified spontaneous emission (ASE) noise is strongly suppressed by another tunable optical filter (filter 2, identical to filter 1). A $1 \mathrm{GHz}$ wide photodetector converts the optical signal into an electric voltage, which is then digitized by a fast oscilloscope. Strain is applied to a $30 \mathrm{~cm}$ segment at the end of a $55 \mathrm{~m}$ sensing fiber. The fiber segment is anchored on one side while its other end is glued to a movable piezoelectric (PZT) stage, which is driven by a sine/triangular wave generator.

To achieve high SR and minimize spatial crosstalk, a short pulse with a high extinction ratio is required. Here we use an RF pulsing scheme [16], in which an EOM modulates the light intensity at a preset high RF frequency, and this modulation is on during the target pulse duration $\tau$. Then a single modulation

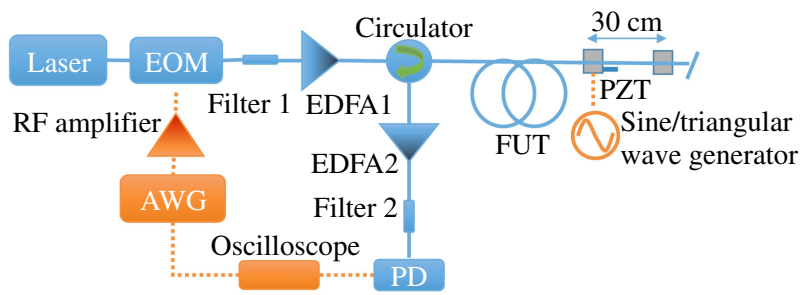

Fig. 2. Schematic of the experimental setup. The AWG helps the generation of short, high extinction ratio pulses, over a precise wide frequency range with close to zero switching latency.

(a)

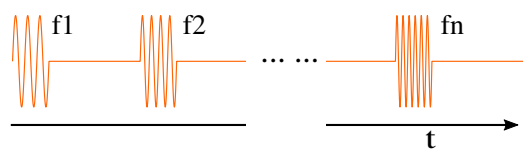

(b)
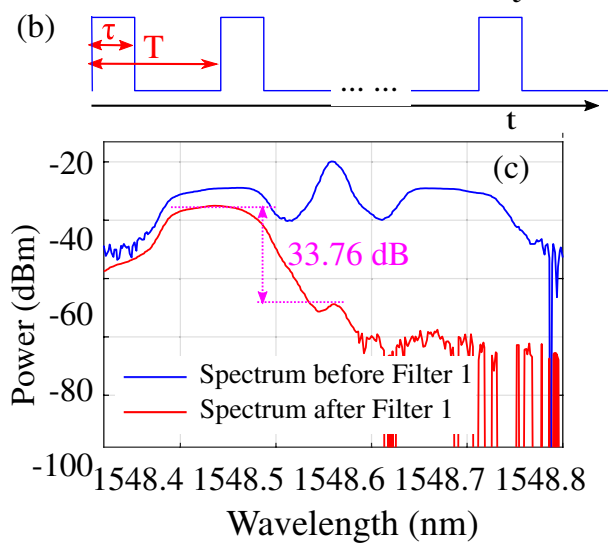

Fig. 3. Schematic diagrams of (a) RF signal applied to the EOM and (b) envelope of the generated pulse train after filter 1; (c) measured spectra of light before and after filter $1(T=0.6 \mu$ s and $\tau=2 \mathrm{~ns})$.

sideband is selected by optically filtering out all other modulation spectral lines, including the optical carrier. The pulse extinction ratio, therefore, depends essentially on the optical filter rejection characteristics rather than on the modulator extinction ratio. This principle is schematically presented in Fig. 3, where a sequence of $N$ RF pulses, spaced $T$ apart and of width $\tau$, each carrying a different RF frequency (from 8 to $20.5 \mathrm{GHz}$ in $50 \mathrm{MHz}$ steps), is derived from the AWG. Unlike conventional frequency scanning, this RF pulse generation technique allows for practically zero latency frequency switching. The schematic diagrams of the RF signal applied to the EOM and the envelope of the generated optical pulse after filter 1 are shown in Figs. 3(a) and 3(b). The measured optical spectra before and after filter 1 are shown in Fig. 3(c) for $T=0.6 \mu$ s, allowing the interrogation of FUTs up to $60 \mathrm{~m}$ long and $\tau=2 \mathrm{~ns}$ ( $20 \mathrm{~cm}$ of spatial resolution). It can be seen that both the carrier and the higher wavelength sideband are highly suppressed. Based on the ratio between the average powers of the filtered sidelobe and the carrier $(33.76 \mathrm{~dB})$ and using the pulse duty cycle of $0.33 \%$, the extinction ratio (the ratio between pulse peak power and carrier power) is determined to be $58.46 \mathrm{~dB}$.

To avoid slowing down the sampling rate with averaging and to still maintain a respectable signal-to-noise ratio (SNR), use was made of an enhanced backscattering fiber [17] (courtesy 


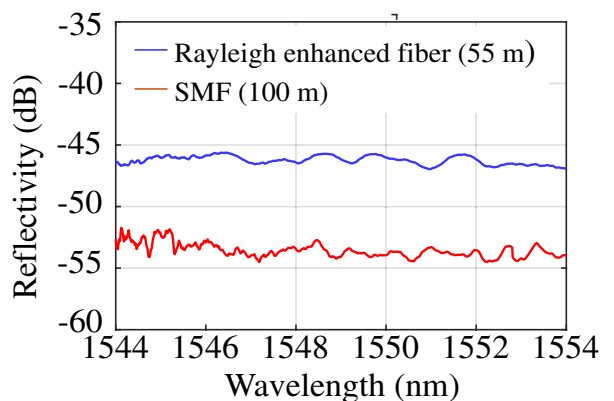

Fig. 4. Enhanced backscattering from the special fiber [17]. The two fibers were illuminated with the ASE from an EDFA and measured with an optical spectrum analyzer using a circulator. The reflectivity here is defined as the reflected spectrum normalized by the input spectrum.

of OFS Ltd), which offers a significantly higher backscattering signal, as compared to a standard single-mode fiber (see Fig. 4).

First, a rather weak $(\sim 2 \mu \varepsilon$ in amplitude), $1 \mathrm{kHz}$ sinusoidal signal was applied to the PZT stage, and the fiber was interrogated with $N=251$ frequency steps, leading to a strain sampling rate of $1 /(T \cdot N)=6.64 \mathrm{kHz}$. The demodulated response from the fiber is shown in the time-distance and frequency-distance maps in Fig. 5. The color scale maps the strain change along the fiber, which is calculated from the frequency shift between each measurement spectra and the reference spectra through cross correlation. Strain variations are clearly visible only at the vibrating segment: about $53.5 \mathrm{~m}$ of distance in the fiber. Figure 6 shows the demodulated signals at $53.5 \mathrm{~m}$ (inside the vibrating segment) and at $54 \mathrm{~m}$ (out of it), again in both domains: (a) time (together with a sinc interpolation) and (b) power spectral density (PSD) (Hanning-weighted periodogram-based PSD). The PSD of the strain in the vibrating section [Fig. 6(b)] reveals a weak harmonic at $2000 \mathrm{~Hz}$. Since no crosstalk is observed in the non-vibrating section, its signal will be treated hereafter as a noise reference, having an average value of $<1.3 \mathrm{n} \varepsilon / \sqrt{\mathrm{Hz}}$ below $700 \mathrm{~Hz}$ and $<0.7 \mathrm{n} \varepsilon / \sqrt{\mathrm{Hz}}$ for higher frequencies, due probably to a larger environmental acoustic noise at lower frequencies.

Figure 7 presents a temporal snapshot of the distribution of strain amplitude around the vibrating fiber segment. The rising edge is $20.3 \mathrm{~cm}$, which is close to the expected spatial resolution from a 2 ns interrogating pulse width.

To further increase the measurement sampling rate by reducing the number of scanning frequencies, $N$, while keeping the same strain coverage (as determined by the frequency scanning range), as well as the frequency step size, we adopt a recently introduced least mean square algorithm that was shown to successfully extend the measurable range of frequency-scanning $\Phi$-OTDRs, while keeping a reduced $N$ [14].

We first apply $N=251$ scanning steps to obtain a reference spectrum for each sensing position with the fiber statically at rest, covering the maximum measurable range offered by the $f_{\max }-f_{\min }=12.5 \mathrm{GHz}$ scanning range. Then, in operation, only $N=60$ frequency scanning steps are used for dynamic measurements, allowing a response time of $36 \mu \mathrm{s}$, which translates to a sampling rate of $27.8 \mathrm{kHz}$. The penalty on the strain uncertainty remains minor $(<1.8 \mathrm{n} \varepsilon / \sqrt{\mathrm{Hz}})$.
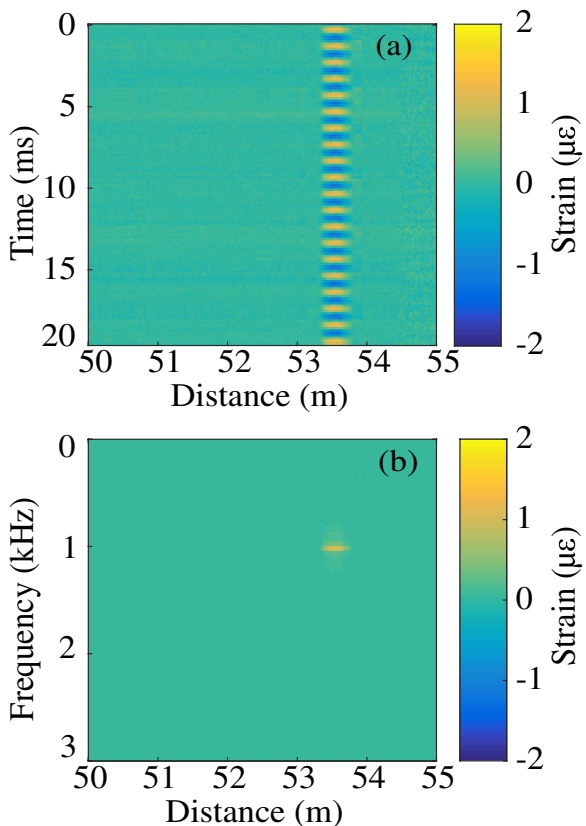

Fig. 5. Strain distribution along the fiber: (a) time domain; (b) frequency domain based on FFT of a $20 \mathrm{~ms}$ long acquisition.
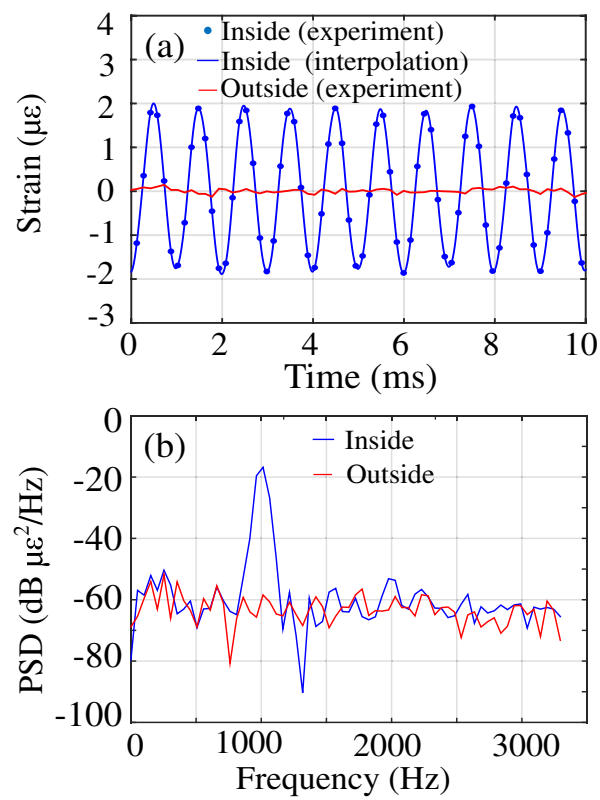

Fig. 6. Demodulated strain inside and outside the vibrating fiber segment: (a) time domain; (b) power spectral density (PSD).

To demonstrate the sensing capability for sizable strain variations, a $100 \mathrm{~Hz}$ triangular voltage signal, generating a peak-to-peak strain variation of $60 \mu \varepsilon$, was applied to the PZT stage, and the measured strain is shown in Fig. 8. The observed deviations from linearity in the rise and fall sections of the recorded signal, Fig. 8(a), are due probably to the way the fiber-loaded stage generated the large, multiple-harmonics dynamic strain signal [Fig. 8(b)]. The maximum stretching range of the PZT prevented us from achieving the maximum strain coverage $>80 \mu \varepsilon$, which is the frequency scanning range 


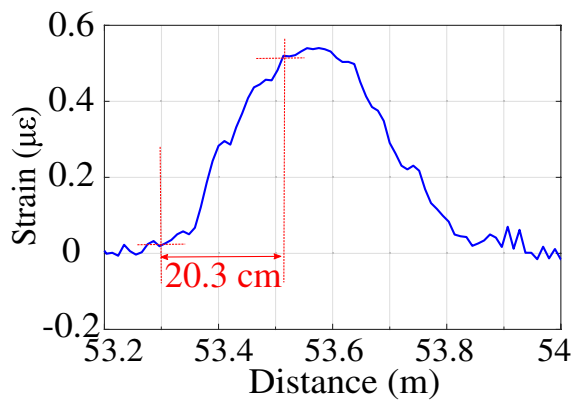

Fig. 7. Instantaneous strain distribution around the vibrating fiber segment, demonstrating the actual $20 \mathrm{~cm}$ spatial resolution.
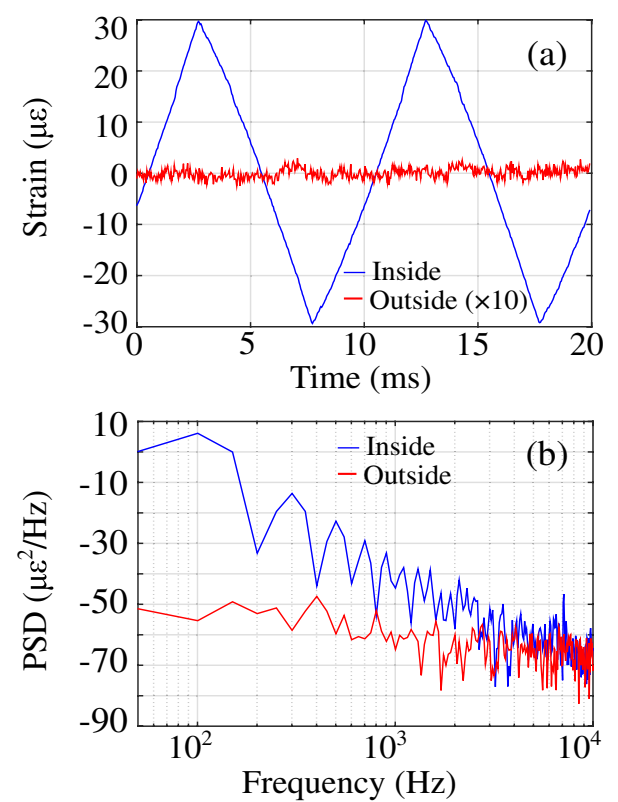

Fig. 8. Demodulated strain for a triangular driving voltage inside and outside the vibrating segment: (a) time domain; (b) PSD.

(12.5 GHz), divided by the strain sensitivity $(150 \mathrm{MHz} / \mu \varepsilon)$ of this fiber. The noise average value is $<1.8 \mathrm{n} \varepsilon / \sqrt{\mathrm{Hz}}$ below $\sim 700 \mathrm{~Hz}$ and $<0.7 \mathrm{n} \varepsilon / \sqrt{\mathrm{Hz}}$ for higher frequencies. The higher uncertainty in the low-frequency range compared with the previous scan scheme, is believed to be due to the environmental noise when acquiring the data. Note that in this technique, the strain-induced frequency shift is estimated by correlating the dynamically varying spectra with a single reference spectrum. This procedure avoids the noise accumulation that accompanies techniques that sum up differential strain changes [18].

While for a longer fiber the maximum sampling rate will decrease, it will still be in the range of hundreds of $\mathrm{Hz}$ (e.g., a $1 \mathrm{~km}$ sensing fiber allows for a sampling rate of $1666 \mathrm{~Hz}$ ).

In conclusion, we demonstrated a simple configuration, capable of detecting fast vibrations and localized faults of sizable strain, such as structural cracks. The proposed solution relies on simple direct detection and performs the function of frequencyscanning $\Phi$-OTDR using RF pulse modulation. The response time could be substantially improved using a backscatterenhanced fiber, further highlighting the large potential of such fibers for distributed sensing [19].
For a direct-detection system, a record high spatial resolution of $20 \mathrm{~cm}$ is demonstrated, concurrently with a relatively large strain measurement range of $60 \mu \varepsilon$. Employing the least mean square algorithm that requires a much restricted set of frequency scanning points, and benefitting from the practically instantaneous frequency switching and no averaging, we achieve a fast measurement sampling rate of $27.8 \mathrm{kHz}$, very close to the maximum possible value of $30 \mathrm{kHz}$ for a $55 \mathrm{~m}$ long sensing fiber with 60 frequency steps. While not used here, real-time strain sensing seems to be technically possible using fast digital signal processors. For applications involving hundreds of meters of fiber, this technique can reliably measure bandwidths of hundreds of Hertz at sub-metric SRs using a robust system compatible with field and on-board tests.

Funding. Horizon 2020 Framework Programme under the Marie Skłodowska-Curie grant "FINESSE" (722509).

Acknowledgment. The authors thank Keysight/Israel for providing the $\mathrm{m} 8195 \mathrm{a}$ arbitrary waveform generator and OFS for the Rayleigh-enhanced optical fiber.

Disclosures. The authors declare no conflicts of interest.

\section{REFERENCES}

1. H. F. Taylor and C. E. Lee, "Apparatus and method for fiber optic intrusion sensing," U.S. patent 5,194,847 (10 October 1993).

2. L. Costa, H. F. Martins, S. Martín-López, M. R. Fernández-Ruiz, and M. González-Herráez, J. Lightwave Technol. 37, 4487 (2019).

3. Y. Wang, Q. Liu, D. Chen, H. Li, and Z. He, IEEE Photon. J. 11, 1 (2019).

4. A. Masoudi, M. Belal, and T. P. Newson, Meas. Sci. Technol. 24, 085204 (2013).

5. G. Fang, T. Xu, S. Feng, and F. Li, J. Lightwave Technol. 33, 2811 (2015).

6. C. Wang, C. Wang, Y. Shang, X. Liu, and G. Peng, Opt. Commun. 346, 172 (2015).

7. J. Xiong, Z. Wang, Y. Wu, and Y. Rao, J. Lightwave Technol. 38, 2028 (2020).

8. Z. Wang, L. Zhang, S. Wang, N. Xue, F. Peng, M. Fan, W. Sun, X. Qian, J. Rao, and Y. Rao, Opt. Express 24, 853 (2016).

9. J. J. Mompó, S. Martín-López, M. González-Herráez, and A. Loayssa, Opt. Lett. 43, 1499 (2018).

10. J. J. Mompó, L. Shiloh, N. Arbel, N. Levanon, A. Loayssa, and A. Eyal, J. Lightwave Technol. 37, 4597 (2019).

11. Y. Koyamada, M. Imahama, K. Kubota, and K. Hogari, J. Lightwave Technol. 27, 1142 (2009).

12. S. Liehr, S. Münzenberger, and K. Krebber, Opt. Express 26, 10573 (2018).

13. J. Pastor-Graells, H. F. Martins, A. Garcia-Ruiz, S. Martin-Lopez, and M. Gonzalez-Herraez, Opt. Express 24, 13121 (2016).

14. L. Zhang, L. D. Costa, Z. Yang, M. A. Soto, M. Gonzalez-Herráez, and L. Thévenaz, J. Lightwave Technol. 37, 4710 (2019).

15. A. K. Sang, M. E. Froggatt, D. K. Gifford, S. T. Kreger, and B. D. Dickerson, IEEE Sens. J. 8, 1375 (2008).

16. J. Urricelqui, A. Zornoza, M. Sagues, and A. Loayssa, Opt. Express 20, 26942 (2012).

17. P. S. Westbrook, K. S. Feder, R. M. Ortiz, T. Kremp, E. M. Monberg, H. Wu, D. A. Simoff, and S. Shenk, 25th Optical Fiber Sensors Conference (OFS) (2017), pp. 1-5.

18. H. D. Bhatta, L. Costa, A. Garcia-Ruiz, M. R. Fernandez-Ruiz, H. F. Martins, M. Tur, and M. Gonzalez-Herraez, J. Lightwave Technol. 37, 4888 (2019).

19. G. Cedilnik, G. Lees, P. E. Schmidt, S. Herstrøm, and T. Geisler, IEEE Sens. Lett. 3, 1 (2019). 